\title{
A Non-Linear Dynamic Model of Ionic Polymer-Metal Composite (IPMC) Cantilever Actuator
}

\author{
D. K. Biswal ${ }^{1 *}$, D. Bandopadhya ${ }^{2}$ and S. K. Dwivedy ${ }^{2}$ \\ ${ }^{1}$ Department of Mechanical Engineering, C V Raman College of Engineering, \\ Bhubaneswar, Odisha-752054, India. \\ *E-mail: dillipkumarbiswal@ gmail.com \\ Phone: +919040450180 \\ ${ }^{2}$ Department of Mechanical Engineering, Indian Institute of Technology Guwahati, \\ Guwahati, Assam-781039, India.
}

\begin{abstract}
This work presents development of an effective non-linear mathematical model for dynamic analysis of Ionic polymer-metal composites (IPMCs) cantilever actuators undergoing large bending deformations under AC excitation voltages. As the IPMC actuator experiences dehydration (solvent loss) in open environment, a model has been proposed to calculate the solvent loss due to applied electric potential following CobbDouglas production method. D'Alembert's principle has been used for the derivation of the governing equation of motion of the system. Generalized Galerkin's method has been followed to reduce the governing equation to the second-order temporal differential equation of motion. Method of multiple scales has been used to solve the non-linear equation of motion of the system and dehydration effect on the vibration response has been demonstrated numerically.
\end{abstract}

Keywords: Ionic polymer-metal composite (IPMC); dehydration Factor; D'Alembert's principle; method of multiple scale.

\section{INTRODUCTION}

Ionic polymer-metal composites (IPMCs) is one of the promising candidates in the field of intelligent materials that received much more attention in recent years both for actuation and sensing related application. When a small external electric potential is applied, IPMC shows considerable bending deformation; conversely a measurable voltage output is obtained across its surface when it is mechanically deformed. These unique properties make them suitable for being used both as actuator and sensor $[1,2]$. In recent years, artificial muscle materials are attracted much more attention as an alternative means of soft actuator or sensor for various industrial and medical applications. Due to their bio-compatibility, large strains at low operating voltage, light in weight and easiness of actuation compared to that of biological systems, electroactive polymers (EAPs) are considered to be a new class of intelligent materials for application anywhere, where a muscle-like response is desirable, including biomedical devices, micro-robotics, aerospace, micro/nano electro-mechanical systems and biomimetic applications [3, 4].

IPMC actuator shows considerable bending vibration, when a fully hydrated IPMC strip was actuated by a small alternative electric potential [5]. An analytical model was developed to show the vibration response of a simply supported IPMC beam 
resting on an elastic foundation subjected to an alternative electric potential [6]. Experimental investigation has been carried out to find out the nonlinear actuation response of cantilever ionic polymer benders and Volterra series has been employed to extract information about the nonlinearity of IPMC actuator [7]. A model has been developed following Hammerstein technique to identify nonlinearity in IPMC materials with the application to walking robots [8]. Nonlinearity in IPMC is caused due to loss of solvent (water) particles in open environment working condition [9]. An analytical model has been proposed to find out the effect of solvent (water) loss on the vibration characteristics of silver-electrode IPMC actuator [10,11]. Considering large bending deformation a nonlinear dynamic model of IPCNC actuators has been proposed using finite element method [12]. An electromechanical model has been developed to predict the mechanical bending deformation of IPMC actuator analytically under DC voltage in a cantilever configuration [13]. A mechanical model has been developed to explain the large bending deformation of IPMC actuator in a cantilever configuration [14]. Based on Euler-Bernoulli beam theory, an equivalent modelling method has been introduced to determine the large nonlinear bending deflection of IPMC actuator in a cantilever configuration [15]. Combining an adaptive neuro fuzzy inference system (ANFIS) and a NARX structure, a dynamic nonlinear black box model has been developed to identify the displacement features of IPMC actuator [16]. Non-uniform curvature of IPMC actuators has been identified by using ANFIS-NARX model [17]. A technique has been developed by using a digital image correlation method to identify the bending strain of IPMC actuators [18].

As the IPMC actuator shows nonlinearity with gradual dehydration and vibration characteristics in a working condition, it is important to study the non-linear vibration characteristics of IPMC actuator and the effect of dehydration on it. Most of the existing works on modelling of IPMC actuators are based on the linear EulerBernoulli beam model [19-22]. Due to the simplicity of these models it cannot take into account large deformation of the IPMC actuators. The model presented in this paper helps to control accurately the tip position of IPMC actuator by compensating the dehydration loss by applying the proportionate input voltage. The objectives of the present analysis include:

i. Investigation and calculation of solvent (water) loss under the application of electric potential.

ii. Analyse and demonstrate the dehydration effect on the non-linear dynamics of an Ag-IPMC actuator under small electric potential.

By considering IPMC actuator as a flexible distributed parameter system a model has been developed and it satisfies Euler-Bernoulli beam assumptions. D'Alembert's principle has been used to develop the governing equation of motion, and the temporal equation of motion has been derived by using generalized Galerkin's method. Method of multiple-scales has been used to solve the temporal equation of motion and subsequently the stability of the system has been studied. The influence of various system parameters on the frequency response curves has also been investigated at resonance condition. Simulations have been performed based on experimental data and by solving the temporal equation of motion of the system. The result obtained by perturbation method and numerically solving the temporal equation of motion shows a good agreement. The paper is organized by first calculating the solvent loss due to applied electric potential, followed by mathematical model for non-linear vibration analysis. Simulation results have been discussed subsequently and finally the conclusion is drawn. 


\section{MATERIALS AND METHODS}

\section{Calculation of Solvent Loss}

An IPMC actuator of silver electrode is prepared and cut into a size of for the experimental study. For fabrication of IPMC actuator Nafion-117 is used as base polymer (thickness of $0.183 \mathrm{~mm}$ ). The Ag-IPMC fabrication followed the traditional scheme composed of surface roughening, adsorption, reduction and developing process, which was proposed in [23]. The dimension of the fabricated IPMC was $45 \mathrm{~mm} \times 25$ $\mathrm{mm} \times 0.2 \mathrm{~mm}$. The photographs of the Nafion-117 membrane and the fabricated Ag IPMC are shown in Figure1 (a) and (b), respectively.

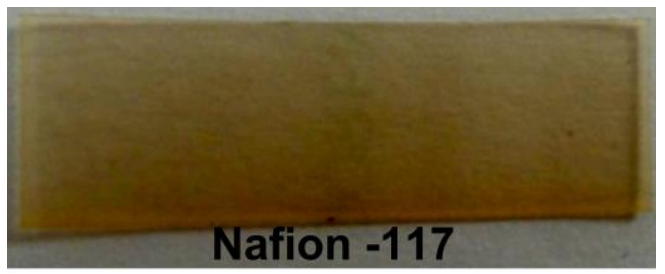

(a)

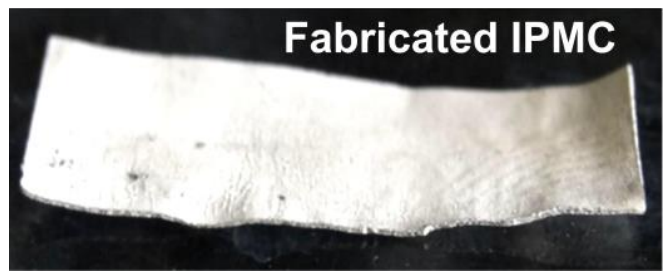

(b)

Figure1. Photograph of the (a) Nafion-117 membrane and (b) fabricated ionic polymermetal composite.

IPMC membranes are made up of an ionic polymer (Nafion or Flemion), which has fixed anions, mobile cations and solvent (water molecules) in the polymer network. When an electric potential is applied across the two surface electrodes, IPMC strip bends due to the electromechanical transduction. Generally, in most of the IPMC actuator water is used as the solvent. The bending response of the IPMC strip depends on the type of polymer base, the mobile cations, and the type of solvent (water). Hence, hydration level is one of the key factors which influence the bending response of the IPMC actuator. In an open environment, the performance of the IPMC actuator is affected by gradual dehydration of water molecules from the IPMC strip. In this study, several experiments are carried out to find out the effect of solvent loss under DC and AC input voltages. The weight of the samples before (dry condition) and after hydration (boiled in distilled water for 30 minutes at $100{ }^{\circ} \mathrm{C}$ ) is measured to calculate the water uptake (WU). All the experiments are conducted in the laboratory, where the temperature and relative humidity was maintained at $26{ }^{\circ} \mathrm{C}$ and $60 \%$. The water uptake of the membrane is the weight ratio of absorbed water inside the wet membrane to that of the dry membrane $[24,25]$. The water uptake value is calculated using the equation shown below:

$W U=\frac{W_{f h}-W_{d r y}}{W_{d r y}}$

where, $W U$ is the water uptake, $W_{f h}$ and $W_{d r y}$ is the weight of the fully hydrated (hydration level is 100\%) and dried IPMC (hydration level is considered to be zero) sample. A fully hydrated IPMC sample is used for conducting experiments to calculate the solvent loss. Both AC and DC voltage is applied for different time periods. A digital 
measuring balance is being used to find out the weight of the sample before and after the experiment. The solvent loss (weight \%) is calculated using the equation (2).

$S_{\text {loss }}=\frac{W_{i}-W_{f}}{M_{w u}} \times 100 \%$

where, $s_{\text {loss }}$ is the solvent loss, $w_{i}$ is the weight of the IPMC sample before the voltage is applied (fully hydrated condition) and $w_{f}$ is the weight of the sample after the voltage is applied for a certain time period. The solvent loss (weight \%) from IPMC actuator for both AC and DC input voltage is shown in Figure 2 and it is observed that solvent loss is much less when the actuator is operated with $\mathrm{AC}$ input compared to DC input voltage. The observation reveals that with $\mathrm{AC}$ input, the actuator can operate for a longer time period than same DC input voltage.

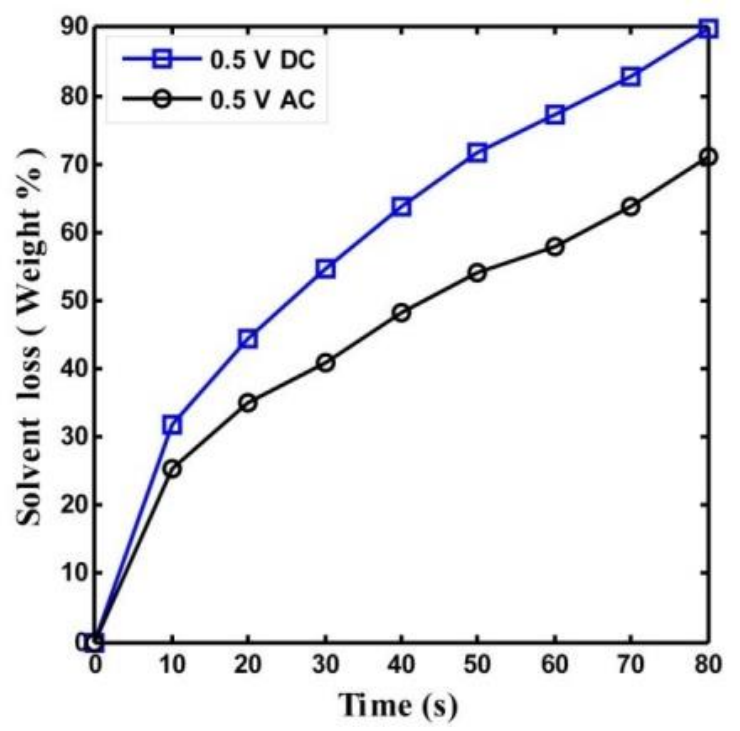

Figure 2. Solvent (water) loss from IPMC with applied voltage.

The relationship between dehydration of water molecules form IPMC strip (lossfactor) in terms of input voltage $(V)$ and time period $(t)$ as given by [10] and can be represented by Eq. (3) is shown in Figure 3.

$\lambda(V, t)=0.9626 V^{0.7184} t^{-0.681}, \quad V \geq 0, t>0$

Where, $\lambda$ is the loss-factor in terms of input voltage $(V)$ and actuation time $(t)$. Figure 3 shows a 3D graph of loss-factor versus input voltage $(V)$ and actuation time $(t)$. These results are in good agreement with the observation that, in an open environment when an input voltage is applied across the surface electrode of an IPMC strip, initial solvent (water) content decreases with actuation time [26]. Further, it is also observed that when electric potential increases, it enhances more water loss from the IPMC strip [27]. From the graph, it is also observed that for a given input voltage, as the hydration level decreases with time there is a gradual reduction of bending deformation (tip deflection) of the IPMC actuator. 


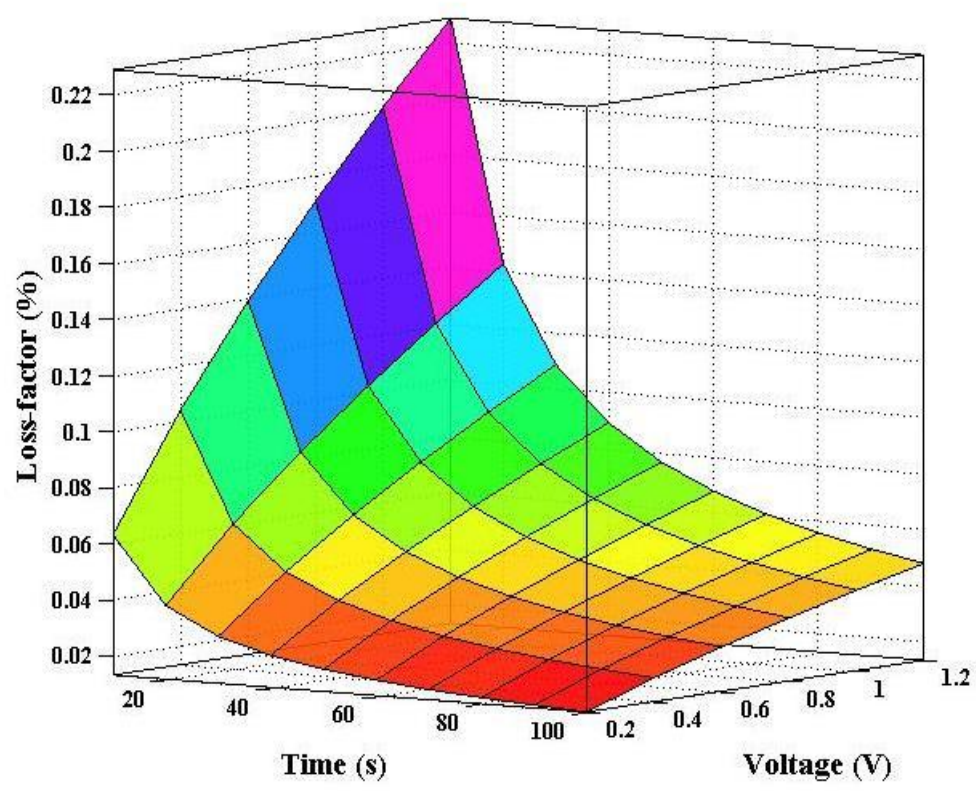

Figure 3. 3D graph of loss-factor versus input voltage and time.

\section{Non-Linear Mathematical Modelling}

Governing equation of motion of a silver-electroded IPMC actuator having length $(L)$, area of cross-section $(A)$, modulus of elasticity $(E)$, density $(\rho)$, and area moment of inertia $(I)$ is developed using d'Almbert's Principle. Figure 4 shows the schematic diagram of the IPMC actuator subjected to an alternating electric potential at its fixed end across its thickness through copper strips in a fixed-free configuration. Mathematical modelling is done by incorporating the tip force generated due to electric potential. Following the Euler-Bernoulli beam theory the actuator is modelled as a uniform flexible distributed parameter system as shown in Figure 4. For an alternating electric potential, the equivalent tip force is assumed to be $F \cos \Omega t$ as shown in Figure 4.

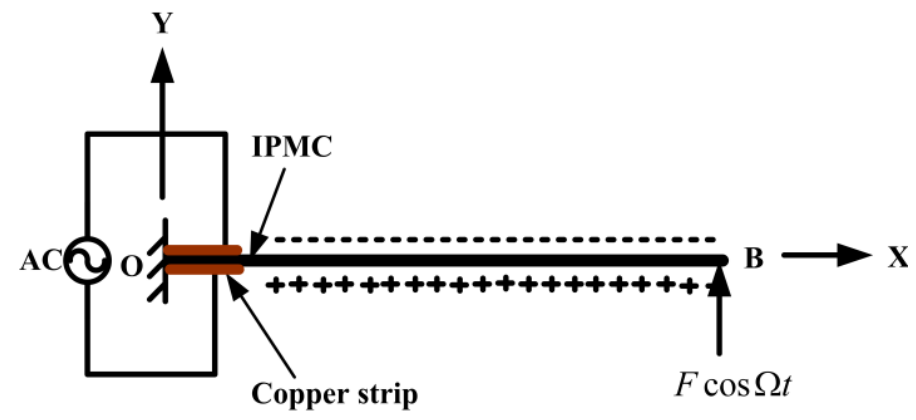

Figure 4. Schematic diagram: un-deformed condition of IPMC actuator.

This equivalent force $(F)$ for 'with' and 'without' dehydration condition can be calculated as follows. Assuming an equivalent force $F$ acting at the free end of the actuator, the maximum bending moment generated by the actuator can be expressed as 
$M=F \times p_{x}$, where $p_{x}$ is the tip position of the actuator in X-coordinate. Considering of pure bending, $\frac{M}{I}=\frac{E}{R_{C}}$, the tip force $(F)$ developed due to input voltage $(V)$ with dehydration and for without dehydration condition can be expressed by Eq. (4) and (5) respectively.

$F_{d}=\frac{E I}{R_{c . d} p_{x}}$

$F_{0}=\frac{E I}{R_{c: 0} p_{x}}$

Where, $R_{c d}$ is the radius of curvature with dehydration and $R_{c 0}$ is the radius of curvature without dehydration.

\section{Temporal equation of motion}

A schematic diagram of the IPMC deformation subjected to alternating electric potential and the coordination system of the actuator are presented in Figure 5. The actuator is operated by applying alternating electric potential at the fixed end and allows it to bend at the free end. The bending moment of the link $M(s)$ at a distance $s$ from the fixed end along the length of the link, as shown in Figure 5 can be expressed as Eq. (6) [28, 29].

$M(s) \approx E I\left[w_{s s}+\frac{1}{2} w_{s}^{2} w_{s s}\right]$

Using the Leibniz's rule and applying the binomial expansion, the equation of motion can be obtained as:

$$
\begin{gathered}
E I\left(w_{s s s s}+\frac{1}{2} w_{s}^{2} w_{s s s s}+3 w_{s} w_{s s} w_{s s s}+w_{s s}^{3}\right)+\rho A w_{s}\left[\int_{0}^{\xi}\left(\dot{w}_{s}^{2}+w_{s} \ddot{w}_{s}\right)\right] d \eta \\
-w_{s s}\left[\int_{s}^{l} \rho A \int_{0}^{\xi}\left(\dot{w}_{s}^{2}+w_{s} \ddot{w}_{s}\right)\right] d \xi d \eta+\left(1-\frac{1}{2} w_{s}^{2}\right)[\rho A \ddot{w}+c \dot{w}] \\
+w_{s} w_{s s}\left[\int_{s}^{l}(\rho A \ddot{w}+c \dot{w}) d \xi\right]=F(1-\lambda) \cos (\Omega t)
\end{gathered}
$$

where, $w$ is the transverse displacement of the link. () is the first derivative with respect to curvature $(s)$ along the link, $\rho$ is the mass density per unit length of the link, $A$ is the cross-section area , $\eta$ and $\xi$ are the variables of integration, $C$ is the damping coefficient, $F$ is the amplitude and $\Omega$ is the frequency of the applied electric potential. 


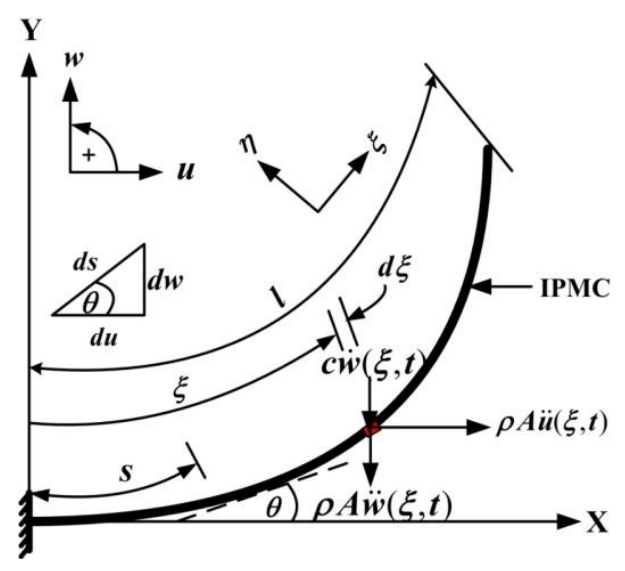

Figure 5. Schematic diagram of a flexible IPMC actuator subjected to bending moment due to input voltage.

Generalized Galerkin's method is used to discretize the governing equation of motion (7) into temporal form by substituting:

$w(s, t)=r \psi(s) q(t)$

where, $r, \psi(s)$, and $q(t)$ are the scaling factor, admissible function and time modulation.

Following [29], here only the single-mode approximation is considered taking into account the predominant fundamental mode.

For further analysis, the following non-dimensional parameters are introduced in this work.

$\bar{w}=\frac{w}{l}, \bar{s}=\frac{s}{l}, \bar{\xi}=\frac{\xi}{l}, \bar{\eta}=\frac{\eta}{l}, \tau=\omega_{1} t, \bar{\Omega}=\frac{\Omega}{\omega_{1}}$

$\omega_{1}$ is the fundamental frequency of the system. Substituting equation (8) and (9) in equation (7) the resulting non-dimensional temporal equation of motion is obtained by applying the generalized Galerkin's method and can be expressed as:

$$
\ddot{q}+q+2 \varepsilon \zeta \dot{q}+\varepsilon \alpha_{1} q^{3}+\varepsilon \alpha_{2} q^{2} \ddot{q}+\varepsilon \alpha_{3} q^{2} \dot{q}+\varepsilon \alpha_{4} q \dot{q}^{2}=\varepsilon \frac{F_{n 0}}{(1-\lambda)} \cos (\bar{\Omega} \tau)
$$

The non-linear temporal equation (10) contains linear forcing term $F_{n}$, cubic geometric term $\alpha_{1} q^{3}$, nonlinear inertia term $\left(\alpha_{2} q^{2} \ddot{q}+\alpha_{4} q \dot{q}^{2}\right)$, and nonlinear damping term $\alpha_{3} q^{2} \dot{q}$. Hence, it is observed that the temporal equation of motion (10) contains many nonlinear terms and it is very difficult to obtain the exact solution. Therefore, following the perturbation technique the solution of the non-linear temporal equation is carried out and thus, method of multiple-scales has been used to solve the equation of motion. 


\section{Solution of temporal equation of motion}

As the closed form solution or exact solution of temporal equation of motion (10) is difficult to obtain, an approximate solution is obtained by using perturbation technique and thus, method of multiple-scales [30] has been used to solve the equation of motion. In this technique the displacement $q$ can be represented in terms of different time scales $\left(T_{0}, T_{1}\right)$ and a book keeping parameter $\varepsilon$ as follows,

$q(\tau ; \varepsilon)=q_{0}\left(T_{0}, T_{1}\right)+\varepsilon q_{1}\left(T_{0}, T_{1}\right)+\varepsilon^{2} q_{2}\left(T_{0}, T_{1}\right)+\ldots \ldots$

where, $T_{0}=\tau, T_{1}=\varepsilon \tau, T_{2}=\varepsilon^{2} \tau \ldots$, and the transformation of the first- and second-time derivatives are obtained as:

$\frac{d}{d \tau}=\frac{d T_{0}}{d \tau} \frac{\partial}{\partial T_{0}}+\frac{d T_{1}}{d \tau} \frac{\partial}{\partial T_{1}}+\ldots \ldots \ldots=D_{0}+\varepsilon D_{1}+\varepsilon^{2} D_{2}+\ldots \ldots$

$\frac{d^{2}}{d \tau^{2}}=D_{0}^{2}+2 \varepsilon D_{0} D_{1}+\varepsilon^{2}\left(D_{1}^{2}+2 D_{0} D_{2}\right)+\ldots$.

Substituting equation (11) and (12) into equation (10) and equating the coefficients of $\varepsilon$ and its power terms, one can obtain the following expressions.

Order $\varepsilon^{0}: D_{0}^{2} q_{0}+q_{0}=0$

Order $\varepsilon^{1}: D_{0}^{2} q_{1}+q_{1}=\frac{F_{n 0}}{(1-\lambda)} \cos \left(\bar{\Omega} T_{0}\right)-2 D_{0} D_{1} q_{0}-2 \xi D_{0} q_{0}$

$$
-\alpha_{1} q_{0}^{3}-\alpha_{2} q_{0}^{2}\left(D_{0}^{2} q_{0}\right)-\alpha_{3} q_{0}^{2}\left(D_{0}^{2} q_{0}\right)-\alpha_{4} q_{0}\left(D_{0} q_{0}\right)^{2}
$$

The general solution of the equation (14) is given by:

$q_{0}=A\left(T_{1}\right) e^{i T o}+\bar{A}\left(T_{1}\right) e^{-i T o}$

Substituting the value of $q_{0}$ from equation (15) into equation (14), and expressing $\cos \left(\bar{\Omega} T_{0}\right)$ in exponential form:

$$
\begin{aligned}
D_{0}^{2} q_{1}+q_{1}= & \left(-2 D_{1} i A-2 \xi i A-3 \alpha_{1} A^{2} \bar{A}+3 \alpha_{2} A^{2} \bar{A}-\alpha_{3} i A^{2} \bar{A}-\alpha_{4} A^{2} \bar{A}\right) e^{i T_{0}} \\
& +\left(-\alpha_{1} A^{3}+\alpha_{2} A^{3}-\alpha_{3} i A^{3}+\alpha_{4} A^{3}\right) e^{3 i T_{0}}+\frac{1}{2} F_{n d} e^{i \bar{\Omega} T_{0}}+c c
\end{aligned}
$$

where $c c$ stands for the complex conjugate of the preceding terms. It is observed that any solution of equation (16) contains both secular and mixed-secular terms when frequency of the electric potential $(\bar{\Omega})$ is nearly equal to 1 . 
Simple resonance case $(\bar{\Omega} \approx 1)$

For the simple resonance case, one may represent detuning parameter $\sigma$ to express the nearness of $\bar{\Omega}$ to 1 , as:

$\bar{\Omega}=(1+\varepsilon \sigma)$, and $\sigma=O(1)$

Eliminating the small divisor terms from equation (16), yields,

$-2 D_{1} i A-2 \zeta i A-3 \alpha_{1} A^{2} \bar{A}+3 \alpha_{2} A^{2} \bar{A}-\alpha_{3} i A^{2} \bar{A}-\alpha_{4} A^{2} \bar{A}+\frac{1}{2} F_{n d} e^{i \sigma T_{1}}=0$

To analyse the solution of equation (18), one may substitute $A=\frac{1}{2} a e^{i \beta}$ where $a$ and $\beta$ are real numbers, in equation (18) and separating the real and imaginary parts of the resulting equation the following expressions are obtained.

$a \gamma^{\prime}=a \sigma-\frac{3}{8} \alpha_{1} a^{3}+\frac{3}{8} \alpha_{2} a^{3}-\frac{1}{8} \alpha_{4} a^{3}+\frac{1}{2} F_{n d} \cos (\gamma)$

$a^{\prime}=-\xi a-\frac{1}{8} \alpha_{3} a^{3}+\frac{1}{2} F_{n d} \sin (\gamma)$

where, $\gamma=\sigma T_{1}-\beta$. For steady-state response $\left(a_{0}, \gamma_{0}\right), a^{\prime}$ and $\gamma^{\prime}$ equal to zero. Eliminating $\gamma$ from equations $(19,20)$, one may find the relation between $\sigma$ and $a$ as:

$\sigma=K a^{2} \pm \sqrt{\left(\frac{F_{n d}}{2 a}\right)^{2}-\left(\xi+\frac{\alpha_{3} a^{2}}{8}\right)^{2}}$

where, $K=\left\{\frac{3 \alpha_{1}}{8}-\frac{3 \alpha_{2}}{8}+\frac{\alpha_{4}}{8}\right\}$. Equation (21) is an implicit form for amplitude of the response as a function of excitation frequency, damping ratio $\xi$ and other system parameters. It is observed that, Eq. (21) does not possess any trivial state response, i.e., $a=0$ is not a solution. The response $(a, \gamma)$ of the system can be obtained by solving Eq. (21) numerically.

\section{RESULTS AND DISCUSSION}

\section{Numerical Results}

Effect of dehydration on the nonlinear vibration response, has been assessed by numerically solving the Eq. (21) the frequency response curve has been obtained for different system parameters. This result has been verified by solving the temporal equation of motion. Results obtained from numerical simulation are based on the physical properties of an IPMC actuator as given in Table 1. The tip deflections data are taken for 30 seconds for each input voltage. It is observed that system exhibits a typical 
nonlinear behavior as various non-linear terms are present in the temporal equation of motion (10).

The non-linear response of the actuator is determined for various applied electric potential. In the following section the responses for primary resonance condition has been discussed. Where, $w_{d}, h, \rho, \varepsilon$ and $r$ are width, thickness, density, book keeping parameter and scaling factor respectively. Figure 6 and Figure 7 demonstrates the frequency response curve with and without dehydration for simple resonance condition for an input of $0.4 \mathrm{~V}$ and $0.8 \mathrm{~V}$ respectively. The stable and unstable responses of the system have been represented by solid and dotted line respectively. It is observed that the system does not possess any trivial state response as shown in the frequency response curves. When the actuator starts to respond at a frequency corresponding to point A (Figure 6), it is observed that with increase in excitation frequency, the response amplitude of the actuator slowly increases. Subsequently, the response amplitude reaches a critical value at point $X$. At this point, with further increase in frequency, the system becomes unstable. Further, increase in frequency drives to the critical point $\mathrm{C}$, beyond which the system again exhibits stable condition.

Table 1 Physical properties of Ag-IPMC actuator.

\begin{tabular}{ccccccc}
\hline$E$ & $L$ & $w_{d}$ & $h$ & $\rho$ & $\varepsilon$ & $r$ \\
\hline $82 \mathrm{MPa}$ & $0.02 \mathrm{~m}$ & $0.005 \mathrm{~m}$ & $0.0002 \mathrm{~m}$ & $2125 \mathrm{~kg} / \mathrm{m}^{3}$ & 0.1 & 0.1 \\
\hline
\end{tabular}

It is further observed that, frequency beyond point $\mathrm{C}$, the amplitude decreases to point $\mathrm{D}$. This observation is consistent with the results presented by [5, 31]. If the system is driven by a voltage with frequency in between point $\mathrm{X}$ and $\mathrm{C}$ the response will be thus unstable and may results in failure in this range. Hence, to operate the system with moderate amplitude of response, either the applied voltage should be lowered, or the operating frequency should be kept well below the bifurcation point $\mathrm{X}$ or above of the bifurcation point $\mathrm{C}$. It is clearly observed that, with dehydration at critical points $\mathrm{B}$ and $\mathrm{C}$, the detuning parameter shifts towards right i.e., the value of detuning parameter increases in case of without dehydration condition. It is further observed that the response amplitude increases in case of without dehydration compared to with dehydration. Similarly, when the excitation frequency is being removed from the system by decreasing in frequency, i.e., from point $\mathrm{D}$, the response amplitude increases and it reaches at point $\mathrm{C}$, which is a saddle node bifurcation point. From this point if the excitation frequency is further increases, the system experiences a jump up phenomenon i.e. a sudden jump from point $C$ to point $B$ which leads to a sudden increase of response amplitude and this may lead to the system failure. 


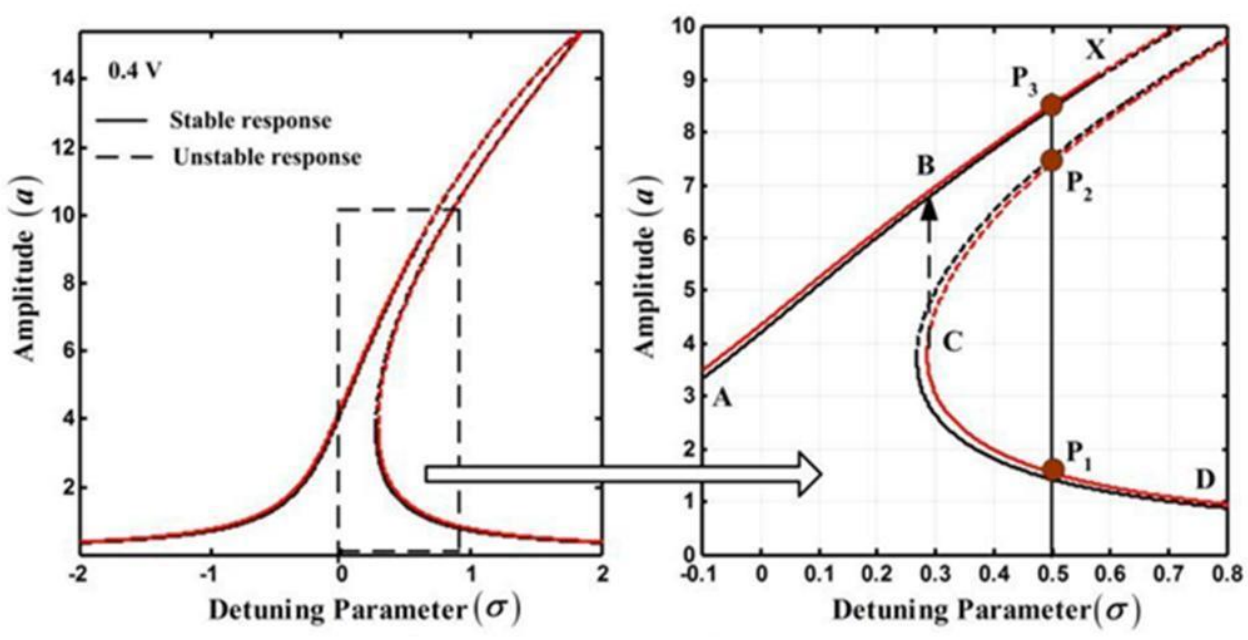

Figure 6. Effect of dehydration on the frequency response curve for $0.4 \mathrm{~V}$.
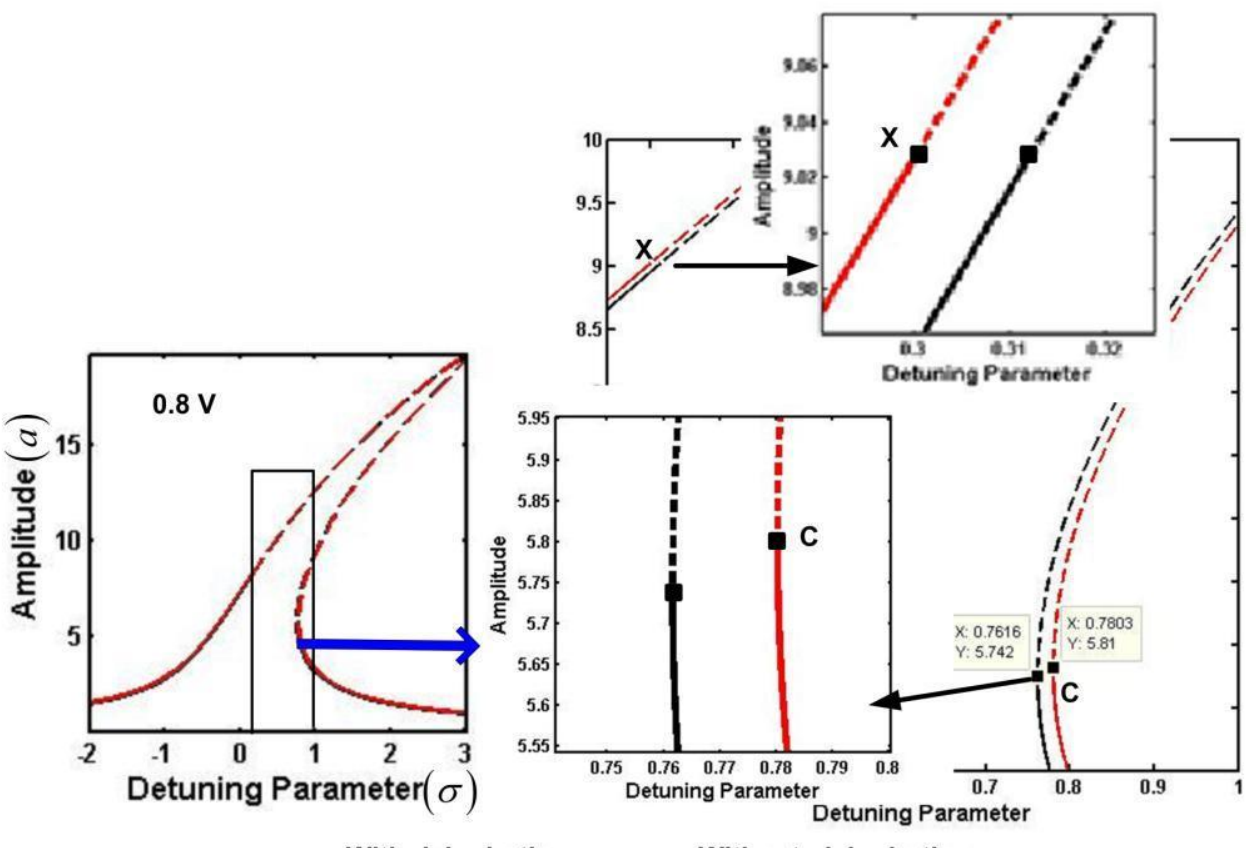

With dehydration

Without dehydration

Figure 7. Effect of dehydration on the frequency response curve for $0.8 \mathrm{~V}$.

Table 2 shows the variation of bifurcation points $\mathrm{C}$ and $\mathrm{B}$, and the jump length for different input voltages for with and without dehydration. It is observed that with increase in the value of excitation voltage, the jump length increases. Comparative study reveals that jump length increases for without dehydration. Table 3 lists the variation of critical points $\mathrm{C}$ and $\mathrm{X}$ with various input voltages for with and without dehydration. Table 3 lists the variation of bifurcation points $\mathrm{C}$ and $\mathrm{X}$ with input voltage. Further, it is clearly observed that the unstable zone, CX increases with increase of input voltage. It is clearly observed that the unstable zone $\mathrm{CX}$ increases as compared to with dehydration condition.

Figure 8 shows the time response and phase portrait, for with and without dehydration condition obtained by solving the Eq. (19) and (20) for an input of $0.4 \mathrm{~V}$. It 
is clearly observed that the response amplitude increases in case of without dehydration condition compared to with dehydration conditions.

Table 2. Variation of the response amplitude $(a)$ at points $\mathrm{C}$ and B with voltage.

\begin{tabular}{lcccccc}
\hline $\begin{array}{l}\text { Voltage } \\
\text { (V) }\end{array}$ & \multicolumn{3}{c}{ Without dehydration } & \multicolumn{3}{c}{ With dehydration } \\
& Point C & Point B & $\begin{array}{c}\text { Jump } \\
\text { length }\end{array}$ & Point C & Point B & $\begin{array}{c}\text { Jump } \\
\text { length }\end{array}$ \\
\hline 0.4 & 3.838 & 6.839 & 3.001 & 3.779 & 6.579 & 2.8 \\
0.6 & 4.823 & 9.372 & 4.549 & 4.747 & 9.21 & 4.463 \\
0.8 & 5.81 & 11.58 & 5.77 & 5.742 & 11.48 & 5.736 \\
1.0 & 6.671 & 13.14 & 6.469 & 6.537 & 12.83 & 6.293 \\
1.2 & 8.034 & 16.07 & 8.036 & 7.989 & 15.75 & 7.761 \\
\hline
\end{tabular}

Table 3. Variation of detuning parameter $(\sigma)$ at critical points of $\mathrm{C}$ and $\mathrm{X}$ with voltage for with and without dehydration.

\begin{tabular}{lllll}
\hline Voltage & \multicolumn{2}{c}{ Without dehydration } & \multicolumn{2}{c}{ With dehydration } \\
(V) & Critical point C & Critical point X & Critical point C & Critical point X \\
\hline 0.4 & 0.2857 & 0.5694 & 0.2861 & 0.5786 \\
0.6 & 0.5133 & 0.4578 & 0.4955 & 0.4701 \\
0.8 & 0.7803 & 0.3075 & 0.7616 & 0.3126 \\
1.0 & 1.007 & 0.1436 & 0.9589 & 0.1899 \\
1.2 & 1.508 & -0.1706 & 1.447 & -0.2079 \\
\hline
\end{tabular}
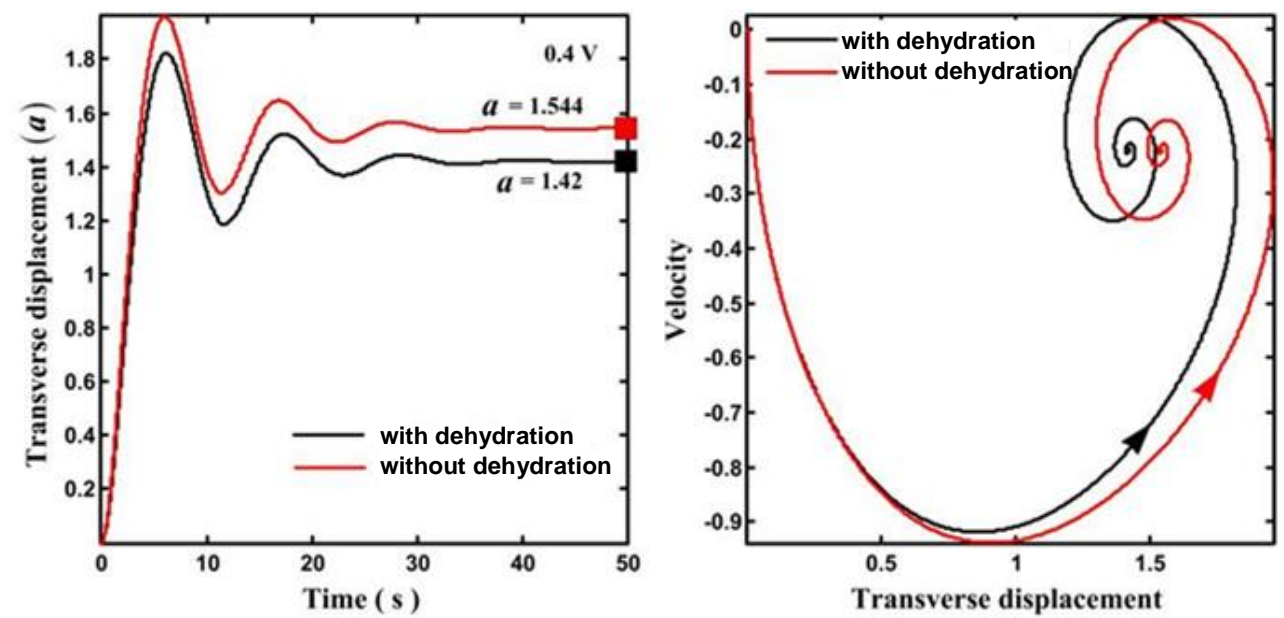

Figure 8. Effect of dehydration on time response and phase portrait curve at $\sigma=0.5,0.4$ V, (Ref: Figure 6).

\section{Experimental Results}

An Ag-IPMC actuator of size $20 \times 5 \times 0.2\left(\mathrm{~mm}^{3}\right)$ is prepared and tested in fixed-free configuration at different applied frequencies under sinusoidal input voltage. Transient and steady-state vibration characteristics are studied and compared with the theoretical results. The photograph of the equipments used in the experimental setup is shown in 
Figure 9. The Rotational Laser Vibrometer (RLV-5500; make: Polytec, GmbH) is connected to one end of the control module and other end of the control module is connected to the computer where the data are processed with the help of Bruel and Kjaer PULSE Lab Shop, Version 13.1.0.246 software. Alternating electric potential is applied to the fixed of the actuator through the function generator (Sony/Tektronix AFG 320) while Laser is directed on tip of the free end. Before the experiment, the fully hydrated IPMC sample is kept in open environment at room temperature for $5 \mathrm{~min}, 10$ min, $15 \mathrm{~min}, 30 \mathrm{~min}$ and $60 \mathrm{~min}$, successively before the experiment to ensure adequate dehydration. Voltage is applied for 30s and subsequently vibration response at the tip of the free end is measured using Rotational Laser Vibrometer. All the experiments are conducted in open environment at room temperature. Figure 10 show the vibration response of the actuator for initial (fully hydrated) condition and after 5 min from the initial condition; after $10 \mathrm{~min}$ and $15 \mathrm{~min}$ and it is observed that peak amplitude reduces considerably due to dehydration. Figure 11 shows unstable response due to vibration of the IPMC actuator for initial and after $10 \mathrm{~min}$ from the initial condition and vibration response amplitude decreases due to dehydration effect.

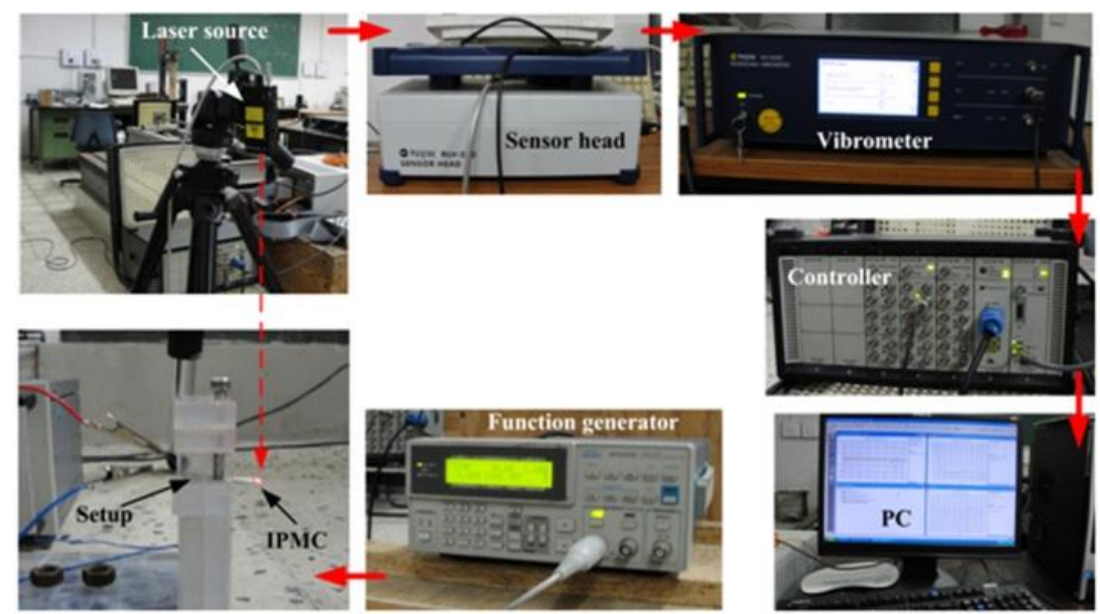

Figure 9. Experimental set-up for bending response analysis.

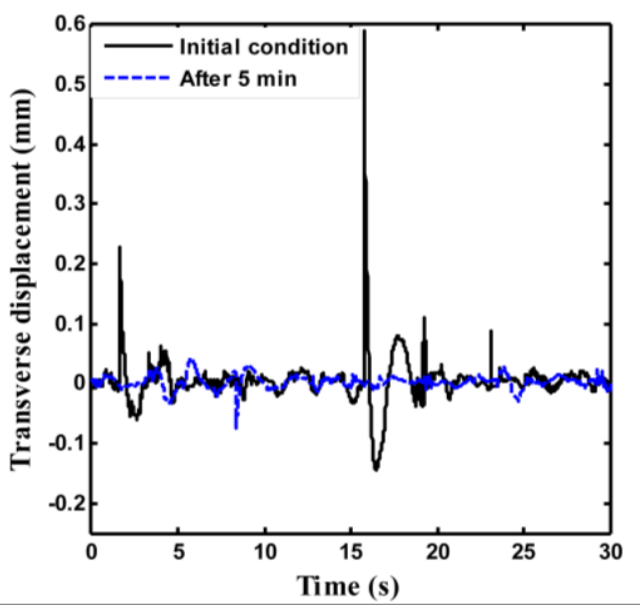

(a)

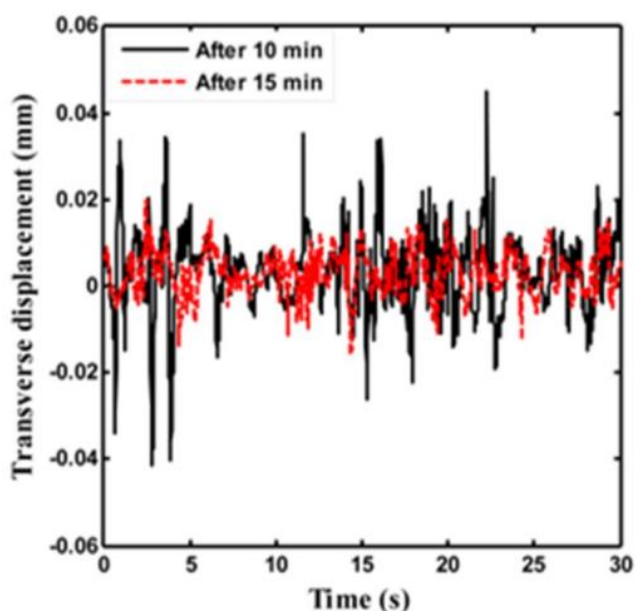

(b)

Figure 10. Experimental vibration response comparison between (a) initial and after 5 min, (b) $10 \mathrm{~min}$ and $15 \mathrm{~min}$ from the starting of the experiment at $0.2 \mathrm{~V}, 1 \mathrm{~Hz}$ sine wave. 


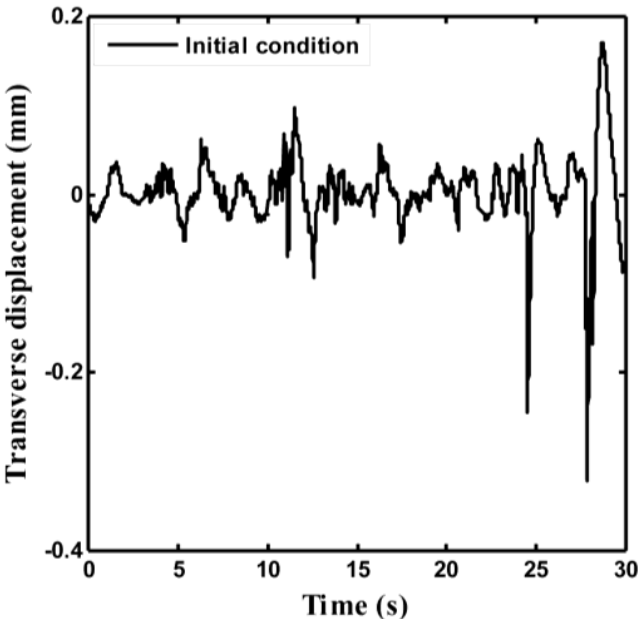

(a)

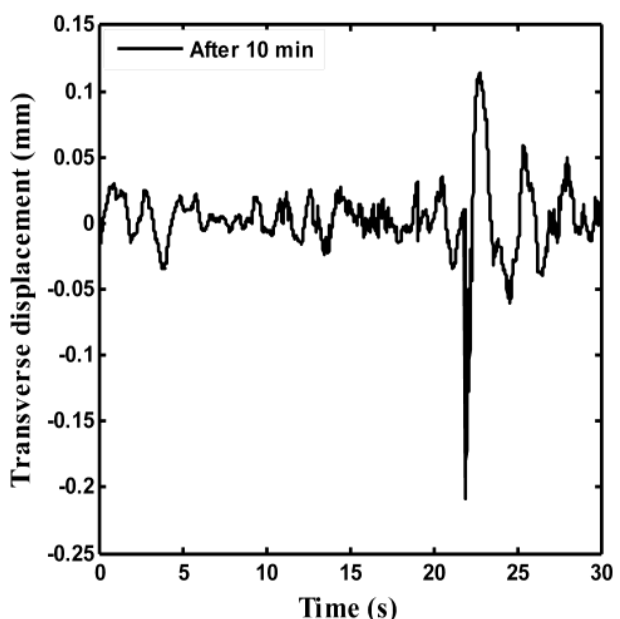

(b)

Figure 11. Experimental vibration response (a) initial condition (b) after $10 \mathrm{~min}$ for an input of $0.6 \mathrm{~V}, 14 \mathrm{~Hz}$ sine wave.

\section{CONCLUSION}

A closed form expression for the amplitude of vibration response and detuning parameter has been derived which can be used to obtain the nonlinear frequency response curve for any IPMC actuator. This simplified expression can be used instead of solving the temporal equation of motion which is time consuming for finding the response of the system. The numerical results are compared and verified by performing experiment. The precise predication of stable and unstable zone for an applied excitation voltage and frequency is of importance in many micromechanical systems utilizing IPMC as the actuator. Both theoretical and experimental investigations confirm that with dehydration, the vibration response amplitude decreases. It is concluded that in micro-scale actuator, amplitude of vibration could increase considerably when a small change in excitation of amplitude occurs. Further, in micro-scale applications, the amplitude of vibration can be easily increased, and it may bring the system into nonlinear (unstable) regime and the system may fail due to that, that why it is necessary to utilize the non-linear analysis to predict the behaviour of the system more accurately.

\section{REFERENCES}

[1] Bar-Cohen Y. Electroactive polymer (EAP) actuators as artificial muscles: reality, potential, and challenges: SPIE press Bellingham, WA; 2004.

[2] Smela E. Conjugated polymer actuators for biomedical applications. Advanced materials. 2003;15:481-94.

[3] Madden JD, Vandesteeg NA, Anquetil PA, Madden PG, Takshi A, Pytel RZ, et al. Artificial muscle technology: physical principles and naval prospects. IEEE Journal of Oceanic Engineering. 2004;29:706-28.

[4] Wallace GG, Teasdale PR, Spinks GM, Kane-Maguire LA. Conductive electroactive polymers: intelligent materials systems: CRC press; 2002.

[5] Sia NN, Yongxian W. Tailoring the actuation of ionic polymer-metal composites. Smart Materials and Structures. 2006;15:909. 
[6] Lei Z, Yaowen Y. Modeling of an ionic polymer-metal composite beam on human tissue. Smart Materials and Structures. 2007;16:S197.

[7] Kothera CS, Leo DJ. Identification of the Nonlinear Response of Ionic Polymer Actuators using the Volterra Series. Journal of Vibration and Control. 2005;11:519-41.

[8] Yamakita M, Kamamichi N, Kaneda Y, Asaka K, Luo ZW. Development of artificial muscle actuator using ionic polymer with its application to biped walking robots. Smart Structures and Materials 2003: Electroactive Polymer Actuators and Devices (EAPAD): International Society for Optics and Photonics; 2003. p. 301-9.

[9] Kothera C, Leo D, Robertson L. Hydration and control assessment of ionic polymer actuators. In 44th AIAA/ASME/ASCE/AHS/ASC Structures, Structural Dynamics, and Materials Conference2003. p. 1441.

[10] Biswal DK, Bandopadhya D, Dwivedy SK. Investigation and evaluation of effect of dehydration on vibration characteristics of silver-electroded ionic polymer-metal composite actuator. Journal of Intelligent Material Systems and Structures. 2013;24:1197-212.

[11] Biswal DK, Bandopadhya D, Dwivedy SK. Dynamic modeling and effect of dehydration on segmented IPMC actuators following variable parameter pseudorigid body modeling technique. Mechanics of Advanced Materials and Structures. 2014;21:129-38.

[12] Moghadam AAA, Hong W, Kouzani A, Kaynak A, Zamani R, Montazami R. Nonlinear dynamic modeling of ionic polymer conductive network composite actuators using rigid finite element method. Sensors and Actuators A: Physical. 2014;217:168-82.

[13] Liu H, Xiong K, Bian K, Zhu K. Experimental study and electromechanical model analysis of the nonlinear deformation behavior of IPMC actuators. Acta Mechanica Sinica. 2017;33:382-93.

[14] Mart A, Alvo A, Andres P, Maarja K. A mechanical model of a non-uniform ionomeric polymer metal composite actuator. Smart Materials and Structures. 2008; 17:025004.

[15] Alici G. An effective modelling approach to estimate nonlinear bending behaviour of cantilever type conducting polymer actuators. Sensors and Actuators B: Chemical. 2009;141:284-92.

[16] Annabestani M, Naghavi N. Nonlinear identification of IPMC actuators based on ANFIS-NARX paradigm. Sensors and Actuators A: Physical. 2014;209:1408 .

[17] Annabestani M, Naghavi N. Non-uniform deformation and curvature identification of ionic polymer metal composite actuators. Journal of Intelligent Material Systems and Structures. 2015;26:582-98.

[18] Punning A, Vunder V, Must I, Johanson U, Anbarjafari G, Aabloo A. In situ scanning electron microscopy study of strains of ionic electroactive polymer actuators. Journal of Intelligent Material Systems and Structures. 2016;27:106174.

[19] Amiri Moghadam AA, Moavenian M, Toussi HE. Modelling and robust control of a soft robot based on conjugated polymer actuators. International Journal of Modelling, Identification and Control. 2011;14:216-26.

[20] Amiri Moghadam AA, Torabi K, Moavenian M, Davoodi R. Dynamic modeling and robust control of an L-shaped microrobot based on fast trilayer polypyrrole- 
bending actuators. Journal of Intelligent Material Systems and Structures. 2013;24:484-98.

[21] Jain RK, Majumder S, Dutta A. SCARA based peg-in-hole assembly using compliant IPMC micro gripper. Robotics and Autonomous Systems. 2013;61:297-311.

[22] Torabi K, Amiri Moghadam AA. Robust control of conjugated polymer actuators considering the spatio-temporal dynamics. Proceedings of the Institution of Mechanical Engineers, Part I: Journal of Systems and Control Engineering. 2012;226:806-22.

[23] Biswal DK, Bandopadhya D, Dwivedy SK. Preparation and experimental investigation of thermo-electro-mechanical behavior of Ag-IPMC actuator. International Journal of Precision Engineering and Manufacturing. 2012;13:77782.

[24] Nemat-Nasser S, Zamani S, Tor Y. Effect of solvents on the chemical and physical properties of ionic polymer-metal composites. Journal of Applied Physics. 2006;99:104902.

[25] Tang Y, Xue Z, Xie X, Zhou X. Ionic polymer-metal composite actuator based on sulfonated poly (ether ether ketone) with different degrees of sulfonation. Sensors and Actuators A: Physical. 2016;238:167-76.

[26] Enikov E, Seo G. Experimental analysis of current and deformation of ionexchange polymer metal composite actuators. Experimental Mechanics. 2005;45:383-91.

[27] Yeh CC, Shih WP. Effects of water content on the actuation performance of ionic polymer-metal composites. Smart Materials and Structures. 2010;19:124007.

[28] Cuvalci O. The effect of detuning parameters on the absorption region for a coupled system: a numerical and experimental study. Journal of Sound and Vibration. 2000;229:837-57.

[29] Zavodney LD, Nayfeh A. The non-linear response of a slender beam carrying a lumped mass to a principal parametric excitation: theory and experiment. International Journal of Non-Linear Mechanics. 1989;24:105-25.

[30] Nayfeh AH, Mook DT. Nonlinear oscillations: John Wiley \& Sons; 2008.

[31] Çilingir HD, Papila M. "Equivalent" Electromechanical Coefficient for IPMC Actuator Design Based on Equivalent Bimorph Beam Theory. Experimental Mechanics. 2010;50:1157-68. 